\title{
The Governance and Its Impact on the Organizational Intelligence from the Point of View for the Employees of the Social Security Corporation (SSC) in Jordan
}

\author{
Eyad Taha Al Rawashdeh ${ }^{1}$ \\ ${ }^{1}$ Business Administration Department, Business Faculty, Tafila Technical University, Tafila, Jordan \\ Correspondence: Dr. Eyad Taha Al - Rawashdeh, Business Faculty, Tafila Technical University, Tafila, Jordan, P. \\ O. Box 179, Tafila, 66110, Jordan. E-mail: dr_eyad71@yahoo.com
}

Received: June 8, 2018

doi:10.5539/ijbm.v13n8p65
Accepted: June 25, 2018

Online Published: June 30, 2018

URL: https://doi.org/10.5539/ijbm.v13n8p65

\begin{abstract}
This study aimed at analyzing the governance and its impact on the organizational intelligence from the point of view of employees of the Social Security Corporation (SSC) in Jordan.

To achieve the objectives of the study, a questionnaire was developed covering the study's variables. The questionnaire was distributed to the entire community of the study, which had 191 managers, deputy directors, heads of department, heads of division as well as employees.

The study used analytical descriptive statistical methods, using the Statistical Package for the Social Sciences (SPSS).

The study reached a number of results, the most important are the following: The general arithmetic mean of the respondents 'perceptions of the dimensions of governance in the SSC came at a medium rating.

Also, the general arithmetic mean of the respondents' perceptions of the dimensions of the organizational intelligence in the SSC came at a medium rating. And, there is a statistically significant relationship between the independent variable (governance) and its different dimensions and the dependent variable (organizational intelligence) and its different dimensions.

In light of these results, the study recommended a number of recommendations, the most important are: establishing a governance system that is declared to all parties concerned to implement it, and clarifies the duties and responsibilities of each employee in the corporation.

Another recommendation is continuing to develop the capabilities that enhance the company's abilities of sensing and responding because of its positive impact on boosting the organizational intelligence.
\end{abstract}

Keywords: governance, organizational intelligence, social security corporation SSC

\section{Introduction}

The current century, its rapid developments and the dramatic changes in various fields of life as a result of the information, cultural and economic revolution and the accompanying transformation in human thought and directions, have made the organizations face many challenges in harmonizing their objectives and how to face these changes.

These organizations resorted to developing modern methods and means, through what management scientists provided, that adapt to the requirements of the modern era. So, the organizations transferred from the traditional management methods to adopt modern methods and strategies produced by scientific progress, such as governance.

Governance is a term that aims at achieving growth, development and economic efficiency and avoiding any upcoming problems and economic crises with the presence of integrity. This concept has emerged in the world in several stages until its development has been completed.

As Jordan is a country that cares about developing its economy in all sectors, It was interested in introducing Governance into organizations due to its significant and important role in developing provided services. 


\subsection{The Problem of the Study}

Over the past decades, there has been a growing interest in corporate governance in many developed and developing economies, especially in the wake of economic collapses and financial crises experienced by a number of countries.

The main reasons for these collapses are organizations management' lack of good practices in controlling and supervision, lack of experience, skill and transparency as well as lack of interest in applying the principles of governance, which is based on applying the principle of transparency and achieving disclosure in presenting information and data that reflect the reality of the financial situation of the organizations as well as other improper practices.

Hence, the problem of the study emerged. The study was interest in studying the Governance and its impact on the intelligence of the organization from the perspective of workers in the SSC in Jordan.

\subsection{Objectives of the Study}

The study aimed at identifying the governance and its impact on the organizational intelligence from the point of view of employees of the SSC through the following sub-goals:

1. Identify the perceptions of the employees of the use of governance in the SSC.

2. Identify the perceptions of the employees of the organizational intelligence in the SSC.

3. Make recommendations and suggestions to decision makers in the SSC based on the results of the study.

\subsection{The importance of the Study}

The importance of the study emerges from addressing a contemporary subject, where governance contributes to raising the level of efficiency and quality of work. The study draws its importance from:

1. The importance of the topic that the study deals with, which is the impact of governance on the organizational intelligence from the point of view of employees in the SSC.

2. Providing a theoretical framework to clarify the concepts of governance' dimensions and the dimensions of the efficiency of administrative decision-making.

3. The role played by the process of using governance to influence public opinion and increasing public confidence in the SSC.

\subsection{Questions of the Study}

To achieve the objectives of this study, the problem emerges from the following main question:

What is the level of governance dimensions on the organizational intelligence in the Social Security Corporation from the point of view of its employees?

The sub-questions emerging from the main question are:

Question 1: What is the level of respondents' perceptions of the concept of governance with its dimensions (participation, transparency and accountability), from the point of view of employees of the S SC?

Question 2: What is the level of the respondents' perceptions about the concept of organizational intelligence with its dimensions (sensing, responding ) from the point of view of employees of the SSC?

\section{Theoretical Framework and Previous Studies}

\subsection{Governance}

Despite the modern use of the term of Governance, its appearance date goes back to a long time. The theoretical and historical basis of the Governance returns first to the agency's theory, which first appeared by the Americans Berls\& Means in 1932.

They noted that there is a separation between the ownership of the company's capital and the process of controlling and supervision within companies, and this separation has its effects on the level of performance of the company (Fateh \& Aishy, 2008).

The definitions presented by researchers on this term have varied according to their different perspectives. The International Finance Corporation (IFC) has defined Governance as the system through which companies are managed and controlled (Alamgir, 2007).

The Organization for Economic Co-operation and Development (OECD) defined it as a set of relationships among the directors of the company, the board of directors, shareholders and other stakeholders (Freeland, 
2007).

In light of the increasing interest in governance, many institutions have studied and analyzed this concept and set specific criteria for its application. These institutions include the Organization for Economic Co-operation and Development, the Bank for International Settlements, represented by the Basel Committee, and the International Finance Corporation of the World Bank.

Corporate governance seeks to protect shareholders' rights, achieve justice, protect the interests of different parties, provide information and integrity of communication channels, and define the enterprise strategy (OECD, 1999)

Many literature has shown that governance is based ,in achieving its objectives, on a set of basic pillars:

1. Disclosure and transparency

2. Accountability

3. Responsibility

4. Justice

5. Independence

6. Laws and regulations

\subsection{The Organizational Intelligence}

The business environment is characterized by constant and unpredictable change which affects the activities of organizations of all kinds. Organizations need to find appropriate solutions to deal with these changes. Organizations that are capable of responding quickly, efficiently, effectively and resilient are able to survive, grow and flourish. And, this is known as the organizational intelligence, which represents the organization's ability to respond to unpredictable, unexpected and sudden environmental changes (Lu \& Ramamurthy, 2011).

(Sambamothy et al., 2003) defined the intelligence as the ability to discover opportunities for depositing in a competitive market by collecting required assets, knowledge and relationships, with the elements of speed and surprise.

The organizational intelligence requires three main capabilities (Sambamothy, 2011):

1. Customer Intelligence: The ability of the organization to know the wishes and preferences of customers.

2. The company's intelligence: The ability of the organization to benefit from the assets, relations and competencies of suppliers, producers and distributors.

3. Operational intelligence: the ability of the organization of work procedures to seize opportunities with speed, accuracy and appropriate cost.

There are different ways in which the organization can build sensing capacity (Trinh, et al., 2012)

1. Deliver information about the surrounding environment to members of the decision-making team, interpret it in a multi-dimensional manner, then analyze the information simultaneously and then group the multiple points of view to come up with a common result, leading to a better sensing process and thus a more intelligent organization.

2. Predictive power: refers to the ability to predict how the market moves, which is a fundamental dimension of the organization's ability of sensing.

Responding capacity: There are four core strategic capacities that can enhance the Organization's response capacity, (Trinh et al. 2012), which are:

1. Developing productive capacities.

2. Developing capacities of the Organization.

3. Adjusting the supply chain and production capacities.

4. Flexibility in the use of resources

\subsection{Previous studies}

Abd Al-ameer and Mohammad, (2017) study, entitled "The Efficiency and Effectiveness of the Internal Audit System and its Impact on the Application of Corporate Governance, Sample of the Iraqi companies listed on the Iraqi market for Securities". The aim of the study was to determine the effectiveness of the internal audit system. It became clear through the study that internal auditing is effective in restructuring the internal control system. 
The study also aimed at demonstrating the role of internal auditing in light of applying the concept of corporate governance. The study reached a number of results, the most important are: demonstrating the effective role of internal auditing in the evaluation of the internal control system and showing the evolution of the concept of internal auditing in light of corporate governance.

The researchers reached that the most important recommendations are: The need to develop the role of internal auditing in a manner that shows how it affects the evaluation of internal control and the need to issue standards for corporate governance

Al-Senoussi (2016) study, entitled "The Impact of Governance on Achieving Competitive Advantage: A Field Study in Private Hospitals in Amman City". The study aimed at identifying the impact of governance in achieving competitive advantage; a field study in private hospitals in Amman. To achieve the objectives of the study, a questionnaire was developed. The study reached a number of results, the most important are: The existence of a statistically significant impact of the application of governance, in all dimensions, combined, in competitive advantage in all dimensions, combined, in private hospitals in Amman.

In light of these results, the study recommended a number of recommendations, the most important is: the need for private hospitals to continue to apply the dimensions of governance.

Al-Tarawneh (2017) study, entitled "External contracting in the employment strategy and its impact on the organizational intelligence, a field study in Jordanian Telecom". The study was conducted to identify external contracting in the employment strategy and its impact on the organizational intelligence in Jordanian telecom companies. To achieve the objectives of the study; a questionnaire was developed to collect data. The study reached a number of results, the most important are: The respondents' perceptions of the dimensions of the independent variable, the external contracting in the employment strategy in its dimensions, have been high. And, the perceptions of the respondents towards the organizational intelligence in its dimensions have been high.

In light of these results, the study recommended the following: The need for companies to make changes and specified policies to the application of external contracting in the employment strategy of Jordanian telecom companies, due to its obvious impact on the organizational intelligence in Jordanian telecom companies.

Abu Baqr (2015) study entitled: "The Impact of the Effectiveness of the Functions of Human Resource Management in the Organizational Intelligence: The Intermediary Role of Human Resources Information Systems, An Empirical Study in Islamic Banks Operating in Jordan". The aim of this study was to identify the impact of the effectiveness of human resources management functions in the organizational intelligence in Islamic banks operating in Jordan. The study has reached a number of results, the most important are : there is a statistically significant impact of human resource management functions on the organizational Intelligence in Islamic banks operating in Jordan, and that there is indirect statistically significant impact of the human resource management functions in the organizational intelligence, through the Human Resources Information Systems of Islamic banks operating in Jordan. This study included two dimensions of organizational intelligence: The intelligence of marketing investment, and the intelligence of operational adaptation.

\section{The Study's Hypotheses}

The first main hypothesis: There is no important, statistical significant impact at the significance level of ( $\alpha \leq$ 0.05 ) of the governance' dimensions (Participation, Transparency, Accountability) on the organizational intelligence' dimensions (sensing, responding) in the SSC.

The following sub-hypothesis emerges:

1. There is no statistically significant impact at the level of $(\alpha \leq 00.05)$ of governance' dimensions (participation, transparency, and accountability) on Sensing as a dimension of organizational intelligence in the SSC.

2. There is no statistically significant impact at the level of $(\alpha \leq 00.05)$ of governance' dimensions (participation, transparency, accountability)on Responding as a dimension of the organizational intelligence in the SSC.

\section{Methodology of the Study}

The study adopted the methodology of descriptive, analytical and field research. On the level of descriptive research, a desk survey was conducted to examine the theoretical and field studies and researches in order to crystallize the foundations and bases of the theoretical framework and to stand at the most important previous studies which constitute a vital contributor to the study because they include axes of knowledge.

On the level of analytical field research, a comprehensive and explorative survey was carried out, and all collected data were analyzed by answering the questionnaires and using the appropriate statistical methods. The study was based on the questionnaire that was developed. 


\subsection{The Study Population}

The study 'community consisted of all 385 employees and managers of the SSC in the capital, and staff number was obtained from the Human Resources Department of the SSC.

\subsection{The Study Sample}

A simple random sample was selected by (49.6), consisting of (191) individuals working in the SSC, Amman Branch, in order to apply the study's instrument to the employees of the corporation. Table No.(1) clarifies the characteristics of the sample of the study.

Table 1. Description of the characteristics of the study sample

\begin{tabular}{llll}
\hline Variable & Category & Number & Percentage \\
\hline Age & 30 years and less & 47 & 24.6 \\
& $31-40$ & 91 & 47.6 \\
& $41-50$ & 47 & 24.6 \\
Gender & 51 years and more & 6 & 3.1 \\
& Male & 122 & 63.9 \\
Qualification & Female & 69 & 36.1 \\
& Second secondary and less & 39 & 20.4 \\
& Community College Diploma & 50 & 26.2 \\
& BA & 90 & 47.1 \\
Career Level & 12 & 6.3 \\
& Post-graduate & 7 & 3.7 \\
& Director & 10 & 5.2 \\
& Deputy Director & 24 & 12.6 \\
& Head of Department & 150 & 78.5 \\
\hline
\end{tabular}

\subsection{The Study's Instrument}

A questionnaire was developed based on the Likert five scale ranging from totally agree and totally disagree with a relative weight (5-1).

The questionnaire was divided into two main groups, the first is concerned with the personal data of the respondents. While the second is concerned with the topic of the research and consists of a group of segments that are related to three basic axes of the independent variable of governance in its dimensions ( participation, transparency and accountability). It will also include a number of paragraphs covering the dependent variable which is the Organizational intelligence: (Sensing, Responding).

\subsection{The Instrument's Reliability}

The questionnaire was presented to a number of arbitrators of faculty members in universities to ascertain the reliability of the questionnaires. Their notes were taken, some paragraphs were reworded, and the required modifications were made to balance the contents of the questionnaire with its paragraphs

\subsection{Stability of the instrument}

The internal consistency coefficient of the study's instrument was extracted using the Cronbach Alpha coefficient for each of the variables of the study in its all dimensions. The values of the stability coefficient were high and indicated the stability and consistency between the paragraphs of the instrument. Table(2) shows the values of the Stability coefficient.

Table 2. The internal consistency coefficient of each variable of the study in its all dimensions

\begin{tabular}{llll}
\hline The Variable & Dimension & Paragraphs & Cronbach Alpha \\
\hline Independent Variable (Governance) & Participation & $1-4$ & 0.753 \\
& Transparency & $5-8$ & 0.762 \\
& Accountability & $9-12$ & 0.781 \\
Dependent Variable & Sensing & $13-16$ & 0.861 \\
(Organizational Intelligence) & Responding & $17-20$ & 0.869 \\
\hline
\end{tabular}


The data in Table 2 shows that the stability coefficients of the dimensions of the governance variable ranged from $(0.753$ to 0.781$)$. As for the organizational intelligence, the stability coefficients ranged between $(0.861$ and $0.869)$. Such values are acceptable for the current study purposes.

\subsection{Statistical Processing}

To answer the questions of the study and to test the validity of its hypotheses, descriptive and analytical statistical methods were adopted, using the statistical package (SPSS.16). The statistical methods used are as follow:

The (Descriptive Statistic Measures) was used to describe the characteristics of the study sample based on frequency and percentages. The Variance Inflation Factory (VIF) and Tolerance Test were used to ensure that there was no high correlation (Multicolinearity) between independent variables. The Skewness test was used to ensure that the data follows normal distributions.

The Multiple regression analysis was used to test the validity of the study models and the effect of the independent variable and its dimensions on the dependent variable and its dimensions. The Stepwise Multiple Regression Analysis was used to test the entry of independent variables in prediction equation of the dependent variable.

\section{The Results}

Before applying the regression analysis to test the hypothesis of the study, some tests were carried out in order to ensure the adequacy of the data for the regression analysis hypotheses. It was ascertained that there is no high correlation between the independent variables (Multicolinearity), using Variance Inflation Factory (VIF) and (Tolerance) test for each variable of the study variables, taking into consideration that the VIF does not exceed the value of (10). And that the Tolerance test value is greater than 0.05 .

It was also ascertained that the data followed the (Normal Distribution) By calculating the (Skewness) coefficient, observing that the data follows the Normal distribution if the Skewness coefficient's value is close to (0). Table (3) shows the results of these tests.

Table 3. Test of the VIF, Tolerance and Skewness

\begin{tabular}{llll}
\hline Independent Variables & VIF & Tolerance & Skewness \\
\hline Participation & 2.097 & 0.477 & -0.360 \\
Transparency & 2.698 & 0.371 & -0.414 \\
Accountability & 2.551 & 0.392 & 0.030 \\
\hline
\end{tabular}

It was noticed that the values of VIF test for all dimensions of the independent variable are less than 10 and range from (2.097 to 2.698). Tolerance test values ranged from ( 0.371 to 0.477$)$, which is an indicator that there is no high correlation (Multicollarity) between the dimensions of the independent variable.

It was ascertained that the data followed the normal distribution by calculating the Skewness coefficient where the values were less than (1). The validity of the model was also validated to test the hypotheses of the study.

The First main Hypothesis: There is no important, statistically significant impact at the level of $(\alpha \leq 0.05)$ for the dimensions of governance (participation, transparency, and accountability) in the organizational intelligence (sensing, responding) in the SSC.

Table 4. The results of multiple regression analysis testing the impact of governance in its various dimensions on the organizational intelligence

\begin{tabular}{llllll}
\hline Governance Dimensions & B & Standard error & Beta & t value & Statistical significance \\
\hline Participation & 0.014 & 0.061 & 0.017 & 0272 & 0.698 \\
Accountability & -0.054 & 0.049 & -0.068 & -1.132 & 0.251 \\
Transparency & 0.116 & 0.059 & 0.149 & $* 2.320$ & 0.026 \\
\hline
\end{tabular}

* is statistically significant at $(\alpha \leq 0.05)$ significance level.

The statistical results in Table 4, following-up on (Beta)coefficients and the (t) test showed that the dimension of governance (transparency) is statistically significant in the dependent variable (organizational intelligence) in 
terms of the rising calculated values of $(t)$,as shown in the previous table at the significance level of $(\alpha \leq 0.05)$, and the statistically significant force of ( Beta) values.

The results in the table above indicated that there is no statistically significant effect of each of the dimensions of governance (participation, accountability) on the dependent variable (intelligence of the organization).

So, there is a statistical significant impact at the level of significance $(\alpha \leq 0.05)$ of the respondents' perceptions of the dimension of governance (transparency) on the organizational intelligence of the SSC.

And, there is a statistically significant impact at the level of significance $(\alpha \leq 0.05)$ of the respondents' perceptions of the dimensions of governance (participation, accountability) on the organizational intelligence of the SSC

Table 5. Results of step wise multiple regression analysis to predict organizational intelligence through governance dimensions

\begin{tabular}{llll}
\hline Entry order of independent variables in the prediction equation & $\mathbf{R}^{\mathbf{2}}$ Value & Calculate $\mathbf{t}$ value & $\mathbf{t}$ significance level \\
\hline Participation & 0.491 & $* \mathbf{1 3 . 2 6 8}$ & 0.000 \\
Accountability & 0.582 & $* \mathbf{5 . 9 9 2}$ & 0.000 \\
Transparency & 0.611 & $* \mathbf{4 . 1 2 7}$ & 0.000 \\
\hline
\end{tabular}

* is statistically significant at $(\alpha \leq 0.05)$ significance level.

The Stepwise multiple regression analysis is used to determine the importance of each one of the independent factor's dimensions separately in contributing to the mathematical model that represents the effect of governance dimensions on organizational intelligence. Table 5 shows the order of entry of independent factor dimensions into the regression equation.

The Participation dimension took the first place and explained (49.1\%) of the variance in the dependent variable (organizational intelligence). It was followed by the Accountability dimension that explained with the previous dimension $(58.2 \%)$ of the variance in the dependent variable (organizational intelligence). Then came the Transparency dimension that explained with the former dimensions $(61.1 \%)$ of variance in the dependent variable (organizational Intelligence).

First sub-Hypothesis: There is no statistically significant impact at the significance level of $(\alpha \leq 00.05)$ of governance in its dimensions (participation, transparency, and accountability) on sensing as a dimension of the organizational intelligence in the SSC.

Table 6. Results of the analysis of variance to verify the validity of the model to test the first sub-hypothesis

\begin{tabular}{lllllll}
\hline Source & R2 coefficient & Total squares & Freedom scores & Squares average & F calculated value & Significance Level of F \\
\hline Regression & & 90.867 & 8 & 11.358 & $* 34.350$ & 0.000 \\
Error & 0.590 & 63.158 & 191 & 0.331 & & \\
Total & & 154.025 & 199 & & & \\
\hline
\end{tabular}

* is statistically significant at $(\alpha \leq 0.05)$ significance level.

The statistical results in Table 6 indicated the validity of the model to test the first sub-hypothesis that there is a statistically significant impact at significance level of $(\alpha \leq 0.05)$ of the respondents' perceptions of the dimensions of governance (participation, transparency and accountability) on sensing as a dimension of organizational intelligence, depending on the calculated value of $(F)$ that reached (34.350) at the significance level $(\alpha=0.000)$ which is significant at the level of significance $(\alpha \leq 0.05)$.

The statistical results in the same table showed that the dimensions of governance as an independent variable explain $(59.0 \%$ ) of the variance in the dependent variable (sensing), which an explanatory force that reflects an acceptable degree of strength and stability of the study model.

Accordingly, we refuse the study's first sub- hypothesis in its nihilistic image, and accept the alternative hypothesis which states: There is a statistically significant effect at the significance level of $(\boldsymbol{\alpha} \leq \mathbf{0 . 0 5})$ of respondents' perceptions of the dimensions of governance (participation, transparency and accountability) on Sensing as a dimension of the organizational intelligence of the SSC. 
Table 7. Results of multiple regression analysis to test the impact of governance and its different dimensions on Sensing

\begin{tabular}{llllll}
\hline Governance Dimensions & B & Standard error & Beta & t value & Statistical significance \\
\hline Participation & 0.023 & 0.071 & 0.019 & 0.319 & 0.750 \\
Transparency & 0.071 & 0.074 & 0.076 & 1.084 & 0.280 \\
Accountability & 0.493 & 0.069 & 0.421 & $* \mathbf{6 . 0 5 1}$ & 0.000 \\
\hline
\end{tabular}

* is statistically significant at $(\alpha \leq 0.05)$ significance level.

The statistical results in Table 7, following up on Beta coefficients and the $(\mathrm{t})$ test showed that the governance dimension (Accountability) is statistically significant in the dependent variable (problem identification) in terms of the rising calculated values of $(t)$, shown in the table at significance level $(\alpha \leq 0.05)$ and the statistically significant influence power of Beta values.

The results in the table above indicated that there is no statistically significant effect of each of the dimensions of governance (participation, transparency) on the dependent variable (sensing). Accordingly, there is a statistical significant impact at the significance level of $(\alpha \leq 0.05)$ for the respondents' perceptions of the dimension of governance (accountability) on sensing as a dimension of the organizational Intelligence in the SSC.

Also, there is no statistically significant effect at the level of significance $(\alpha \leq 0.05)$ of the respondents' perceptions of the dimensions of governance (participation, transparency) on sensing as a dimension of the organizational intelligence in the SSC.

The Second Sub-Hypothesis: There is no statistically significant impact at the level of significance of ( $\alpha \leq$ 0.05) of governance in its dimensions (participation, transparency, and accountability) on Responding as a dimension of the organizational intelligence in the SSC.

Table 8. Results of Analysis of Variance to verify the validity of the model to test the second sub-hypothesis

\begin{tabular}{lllllll}
\hline Source & R2 coefficient & Total squares & Freedom degrees & $\begin{array}{l}\text { Squares } \\
\text { average }\end{array}$ & Calculated F value & F significance level \\
\hline Regression & & 82.269 & 8 & 10.284 & $* \mathbf{3 0 . 0 1 1}$ & 0.000 \\
error & 0.557 & 65.449 & 191 & 0.343 & & \\
Total & & 147.718 & 199 & & & \\
\hline
\end{tabular}

$*$ is statistically significant at $(\alpha \leq 0.05)$ significance level.

The statistical results in Table 8 indicated the validity of the model to test the second sub-hypothesis and that there is a statistically significant effect at the significance level of $(\alpha \leq 0.05)$ of the respondents' perceptions of the dimensions of governance (participation, transparency and accountability) on responding as a dimension of the organizational intelligence, based on the calculated value of $(F)$ which reached (30.011) at the significance level $(\alpha=0.000)$ which is significant at the level of significance $(\alpha \leq 0.05)$.

The statistical results in the same table showed that the dimensions of governance as an independent variable explain (55.7\%) of variance in the dependent variable (responding), which is an explanatory force that reflects an acceptable degree of strength and stability of the study model.

Accordingly, we reject the second sub-study as nihilistic, and accept the alternative hypothesis, which states that there is a statistically significant impact at the significance level $(\alpha \leq 0.05)$ of respondents' perceptions of the dimensions of governance (participation, transparency and accountability) in the alternative test as a dimension of the organizational intelligence in the SSC.

Table 9. The results of multiple regression analysis to test the impact of governance and its various dimensions on Responding

\begin{tabular}{llllll}
\hline Governance Dimensions & B & Standard error & Beta & t value & Statistical significance \\
\hline Participation & 0.032 & 0.075 & 0.030 & 0.426 & 0.671 \\
Transparency & -0.029 & 0.081 & -0.029 & -0.364 & 0.716 \\
Accountability & 0.298 & 0.081 & 0.287 & $* \mathbf{3 . 6 6 4}$ & 0.000 \\
\hline
\end{tabular}

* is statistically significant at $(\alpha \leq 0.05)$ significance level. 
The statistical results in Table 9, following-up on Beta coefficients and the (t) test showed that governance dimension (Accountability) is statistically significant in the dependent variable ( Responding) in terms of rising calculated value of $(t)$ as shown in the previous table at the significance level of $(\alpha \leq 0.05)$ and the statistically significant influential power of Beta values.

The results in the table above also indicated that there is no statistically significant effect of each of the governance dimensions (participation and transparency) on the dependent variable (responding). Accordingly, there is a statistically significant impact at the significance level $(\alpha \leq 0.05)$ for respondents' perceptions of the dimension of governance (accountability) in responding as a dimension of the organizational intelligence in the SSC.

Also, there is no statistically significant effect at the significance level $(\alpha \leq 0.05)$ for respondents' perceptions of the dimensions of governance (participation and transparency) on responding as a dimension of the organizational intelligence in the SSC.

\section{Discussion of Results}

1. The results showed that the general arithmetic mean of the respondents' perceptions of the dimensions of governance in the SSC came at a medium rate.

2. The results showed that the general arithmetic mean of the respondents' perceptions of the dimensions of the organizational intelligence in the SSC came at a medium rate

3. The results indicated that there is a statistically significant positive relationship between the independent variable (governance) and its different dimensions and the dependent variable (organizational intelligence) and its different dimensions

4. The results also indicated a statistically significant effect at the significance level of $(\boldsymbol{\alpha} \leq \mathbf{0 . 0 5})$ for respondents' perceptions of the dimensions of governance, combined, (transparency, accountability, participation) on the organizational intelligence in the SSC.

\section{Recommendations}

1. Spreading and promotion the culture of governance among employees, as this will reflect on the decisions of the corporation, and will be positively reflected on its performance.

2. Establishing a system of governance announced to all parties concerned to implement it, clarifying the duties and responsibilities of each employee in the corporation.

3. Start the effective implementation of the principles of governance to reduce administrative corruption.

4. Continuing to develop the enhancing capabilities of companies in sensing and responding due to the positive impact on boosting the organizational intelligence.

\section{References}

Abu Baqr, M. (2015). The Impact of the Effectiveness of Human Resource Management Functions in the Organizational Intelligence: The Simple Role of Human Resources Information Systems, An Applied Study in Islamic Banks Operating in Jordan. PhD thesis, International Islamic Sciences University, Jordan

Alamgir, M. (2007). Corporate Governance: A Risk Perspective, paper presented to: Coorporate Governance and Reform: Paving the Way to Financial Stability and Development, a conference organized by the Egyptian Banking Institute, Cairo.

Ali, N. A. A., \& Ali, K. M. (2017). Efficiency and Effectiveness of the Internal Audit System and its Impact on the Application of Corporate Governance. Sample of Iraqi Companies Listed in the Iraqi Stock Exchange. Al-Muthanna Journal of Administrative and Economic Sciences, 7(4).

Freeland, C. (2007). Basel Committee Guidance on Corporate Governance for Banks, paper presented to: Corporate Governance and Reform: Paving the Way to Financial Stability and Development, a conference organized by the Egyptian Banking Institute, Cairo.

Lu, Y., \& Ramamurthy, K. (2012). Understanding the link between information technology capability and organizational agility: An empirical examination. Pfeiffer university, 35(4), 931-935.

OECD. (1999). OECD Principles of Corporate Governance.

Sambamurthy, V., \& Bharadwaji, A. (2003). Shaping Agility Through Digital Options: Reconcepualizing the Role of Informationms Technology in Contemporary Firms. University of Mnnesota, 27(2), 237-263. 
Sinousi, I. W. (2016). The Impact of Governance on Achieving Competitive Advantage. A Field Study in Private Hospitals in Amman, Unpublished Master Thesis, Middle East University, Jordan

Tarawneh, B. M. (2017). Outsourcing in the Employment Strategy and its Impact on the Intelligence of the Organization A Field Study in Jordanian Communication. Unpublished Master Thesis, Mutah University, Jordan.

Triguero, R., Vinces, J., Rendon, M., \& Apellaniz, M. (2012). Human Resource Management Practices Aimed at Seeking the Commitment of Employees on Financial and non-finacial (subjective) Performance in Spnish Firms: An Empirical Contribution.

\section{Copyrights}

Copyright for this article is retained by the author(s), with first publication rights granted to the journal.

This is an open-access article distributed under the terms and conditions of the Creative Commons Attribution license (http://creativecommons.org/licenses/by/4.0/). 